\title{
Dans quels pays et établissements les élèves défavorisés réussissent-ils ?
}

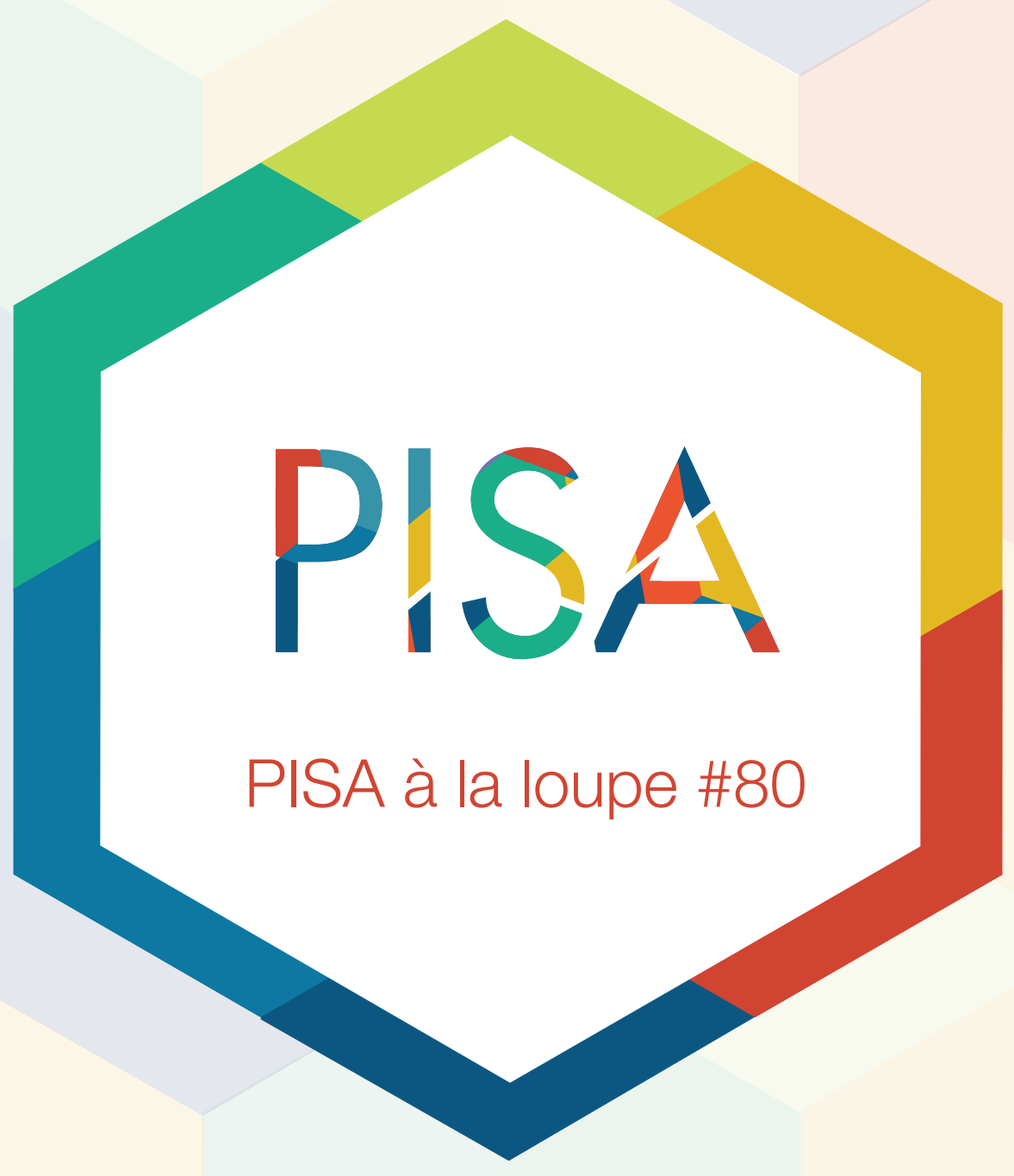




\section{Dans quels pays et établissements les élèves défavorisés réussissent-ils ?}

- Bien que comptant parmi les 25 \% les plus pauvres de son pays, un élève défavorisé sur quatre est résilient sur le plan scolaire et atteint au moins le niveau 3 de compétence dans l'ensemble des trois principaux domaines d'évaluation PISA.

- En Allemagne, au Canada, en Corée, au Danemark, en Estonie, en Finlande, à Hong-Kong (Chine), en Irlande, au Japon, en Norvège, aux Pays-Bas, à Singapour, en Slovénie et au Viet Nam, plus de 30 \% des élèves de 15 ans issus d'un milieu défavorisé sont résilients sur le plan scolaire.

- Dans la grande majorité des systèmes d'éducation, les établissements où les élèves ont les meilleures chances d'être résilients sur le plan scolaire présentent certaines caractéristiques communes - au premier rang desquelles un bon climat de discipline, qui permet aux élèves de se concentrer en classe et aux professeurs de dispenser leur enseignement au rythme qu'ils souhaitent.

La plupart des élèves peu performants dans l'enquête PISA sont issus d'un milieu socio-économique défavorisé. Toutefois, parmi leurs pairs défavorisés, certains surmontent les obstacles qui entravent leur parcours et excellent aux évaluations PISA.

\section{Certains pays parviennent mieux que d'autres à aider les élèves les plus défavorisés à réussir à l'école.}

D'après les données de l'enquête PISA, en moyenne, dans les pays de l'OCDE, trois élèves sur quatre se situant dans le quartile inférieur du statut socio-économique n'atteignent au mieux que le niveau de compétence de base (niveau 2) en compréhension de l'écrit, en mathématiques ou en sciences. Alors qu'en Allemagne, au Canada, en Corée, au Danemark, en Estonie, en Finlande, à Hong-Kong (Chine), en Irlande, au Japon, en Norvège, aux Pays-Bas, à Singapour, en Slovénie et au Viet Nam, plus de 30 \% des élèves défavorisés atteignent au moins le niveau 3 de compétence dans l'ensemble des domaines d'évaluation de l'enquête PISA 2015 - et peuvent donc être considérés comme « résilients sur le plan scolaire » -, en Algérie, au Kosovo, au Pérou, en République dominicaine et en Tunisie, moins de $1 \%$ des élèves défavorisés satisfaisant les conditions pour passer les évaluations PISA 2015 parviennent à ce niveau.

Au niveau 3 de compétence, les élèves commencent à faire preuve de capacités à : dégager la signification d'un texte et parvenir à une compréhension détaillée à partir de multiples fragments indépendants d'information en compréhension de l'écrit ; établir des relations proportionnelles et se livrer à une interprétation et un raisonnement simples dans le cadre de la résolution d'un problème mathématique ; et appréhender des sujets qui ne leur sont pas familiers en sciences autant de compétences au fondement de la réussite et de la poursuite de l'apprentissage tout au long de la vie.

La variation du pourcentage d'élèves résilients sur le plan scolaire entre les pays et dans le temps reflète en grande partie les différences de performance moyenne de l'ensemble des élèves. Les pourcentages les plus faibles d'élèves résilients s'observent ainsi dans les pays où la performance moyenne est faible, même parmi les élèves plus favorisés. Toutefois, cette variation reflète également des disparités dans l'équité de la répartition des possibilités d'apprentissage. Ainsi, au Danemark et en Suisse, 49 \% environ des élèves atteignent au moins le niveau 3 de compétence, mais l'association entre le statut socio-économique et la performance est significativement plus forte en Suisse et le pourcentage d'élèves résilients y est en conséquence significativement plus faible qu'au Danemark.

D'après les données collectées sur une période de dix ans dans le cadre de l'enquête PISA (en 2006, 2009, 2012 et 2015), plusieurs pays sont parvenus à accroître le pourcentage d'élèves résilients sur le plan scolaire parmi ceux se situant dans le quartile inférieur du statut socio-économique. Parmi les 51 systèmes d'éducation permettant la comparaison du pourcentage d'élèves résilients entre 2006 et 2015, 19 ont vu leur pourcentage d'élèves défavorisés résilients augmenter, tandis que 9 l'ont vu diminuer. Parmi les pays de l'OCDE, cette hausse est particulièrement marquée en Allemagne, en Espagne, en Israël, au Japon, en Norvège, en Pologne, au Portugal et en Slovénie. Ainsi, en Allemagne, seul environ un élève défavorisé sur quatre atteignait au moins le niveau 3 de compétence dans l'ensemble des trois domaines d'évaluation PISA en 2006, contre un sur trois en 2015. À l'inverse, en Australie, en Corée, en Finlande, en Hongrie, en Nouvelle-Zélande et en Suède, le pourcentage de ces élèves a diminué au cours de la même période. En Finlande, près de 56 \% des élèves défavorisés étaient résilients en 2006, contre seulement 39 \% en 2015. 
Combien d'élèves défavorisés réussissent dans l'enquête PISA ?

Pourcentage d'élèves se situant dans le quartile inférieur du statut socio-économique qui atteignent au moins le niveau 3 de compétence en compréhension de l'écrit, en mathématiques et en sciences

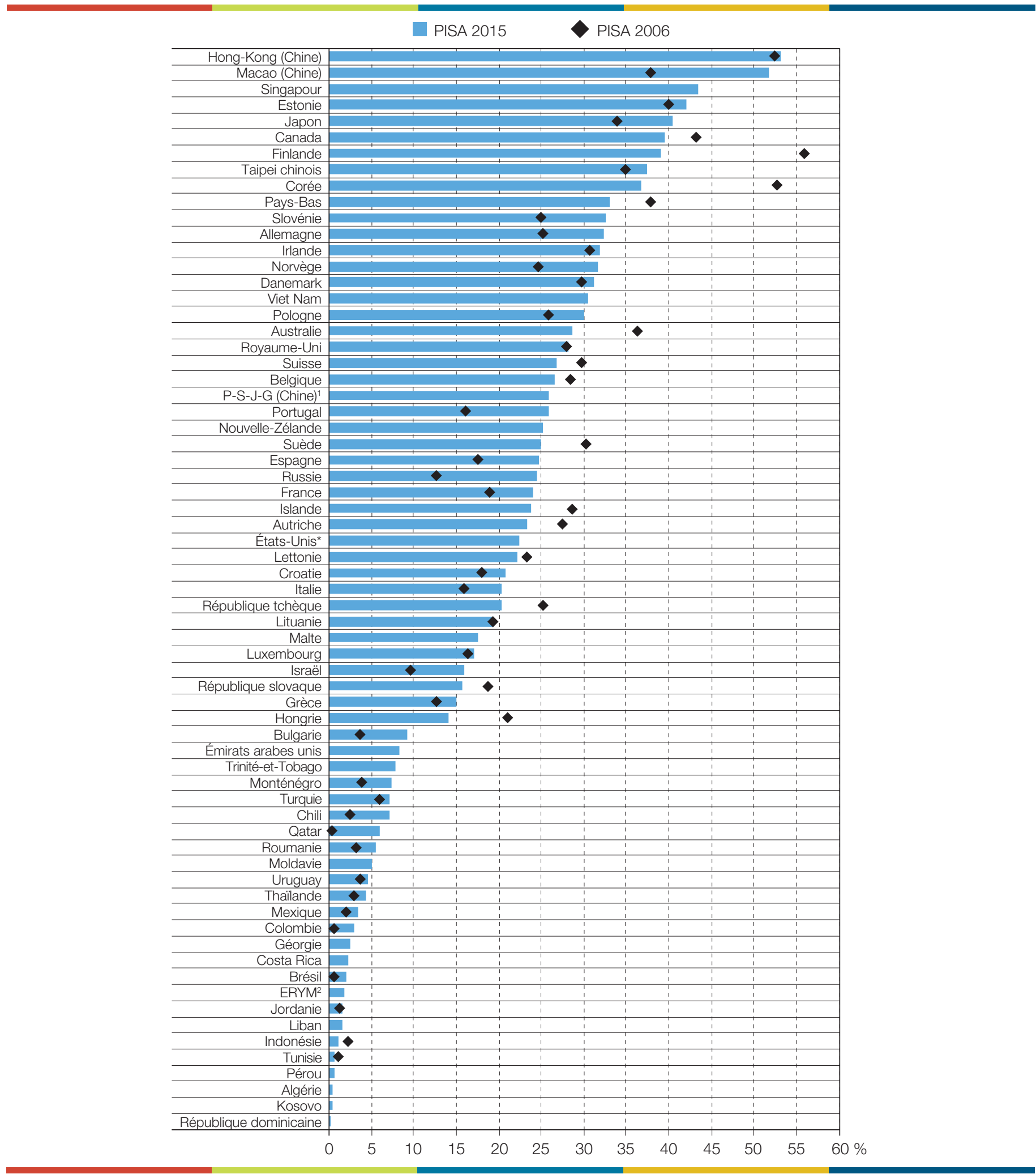

1. P-S-J-G (Chine) désigne les quatre provinces chinoises participant à l'enquête PISA : Pékin, Shanghai, Jiangsu et Guangdong.

2. ERYM est l'acronyme utilisé pour l'ex-République yougoslave de Macédoine.

Remarques : Les résultats de l'enquête PISA 2006 sont manquants pour les pays qui n'ont pas participé à cette évaluation.

* Les résultats de l'évaluation PISA 2006 de la compréhension de l'écrit ne sont pas disponibles pour les États-Unis.

Les pays et économies sont classés par ordre décroissant du pourcentage d'élèves défavorisés résilients sur le plan scolaire dans l'enquête PISA 2015.

Source : OCDE, Bases de données PISA 2006 et PISA 2015. 


\section{Facteurs scolaires associés à la résilience des élèves}

Parmi les élèves défavorisés, il existe une relation positive/négative entre la probabilité d'atteindre au moins le niveau 3 de compétence en compréhension de l'écrit, en mathématiques et en sciences, et :

\begin{tabular}{|c|c|c|c|c|c|}
\hline & \multicolumn{2}{|c|}{ NS Relation non significative } & Relation positive & \multicolumn{2}{|l|}{ Relation négative } \\
\hline & \multicolumn{3}{|c|}{ Ressources de l'établissement } & \multicolumn{2}{|c|}{ Climat de l'établissement } \\
\hline & $\begin{array}{l}\text { Taille moyenne d'une classe } \\
\text { de la langue d'enseignement }\end{array}$ & $\begin{array}{c}\text { Ratio entre le nombre } \\
\text { d'ordinateurs mis à } \\
\text { disposition des élèves et } \\
\text { le nombre d'élèves dans le } \\
\text { grade modal pour les élèves } \\
\text { de } 15 \text { ans }\end{array}$ & $\begin{array}{l}\text { Nombre d'activités } \\
\text { extrascolaires proposées } \\
\text { dans l'établissement }\end{array}$ & $\begin{array}{c}\text { Pourcentage d'élèves qui } \\
\text { n'avaient séché aucune } \\
\text { journée de cours durant les } \\
\text { deux semaines précédant } \\
\text { l'évaluation PISA }\end{array}$ & $\begin{array}{c}\text { Climat en classe propice } \\
\text { à l'apprentissage }\end{array}$ \\
\hline Moyenne OCDE & & NS & & & \\
\hline \multicolumn{6}{|l|}{ OCDE } \\
\hline Australie & NS & NS & NS & NS & \\
\hline Autriche & NS & NS & & NS & \\
\hline Belgique & & NS & & & \\
\hline Canada & & NS & & & \\
\hline Chili & NS & NS & NS & & \\
\hline République tchèque & & NS & NS & & \\
\hline Danemark & NS & NS & NS & & \\
\hline Estonie & NS & NS & NS & & \\
\hline Finlande & NS & NS & NS & NS & NS \\
\hline France & NS & NS & NS & NS & NS \\
\hline Allemagne & NS & NS & & NS & \\
\hline Grèce & NS & & NS & 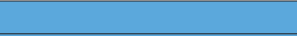 & \\
\hline Hongrie & NS & NS & & & \\
\hline Islande & NS & NS & NS & NS & NS \\
\hline Irlande & & NS & NS & NS & \\
\hline Israël & NS & & & NS & \\
\hline Italie & NS & NS & NS & & \\
\hline Japon & & NS & & NS & \\
\hline Corée & NS & & & & \\
\hline Lettonie & NS & NS & NS & & \\
\hline Luxembourg & NS & & NS & NS & NS \\
\hline Pays-Bas & & NS & NS & & \\
\hline Nouvelle-Zélande & NS & & & & NS \\
\hline Norvège & NS & NS & NS & & \\
\hline Pologne & NS & NS & NS & & NS \\
\hline Portugal & & NS & NS & & \\
\hline République slovaque & & NS & NS & NS & \\
\hline Slovénie & & & NS & & \\
\hline Espagne & NS & NS & NS & & \\
\hline Suède & NS & NS & NS & & NS \\
\hline Suisse & NS & & NS & & \\
\hline Turquie & NS & NS & NS & NS & \\
\hline Royaume-Uni & & NS & NS & NS & \\
\hline États-Unis & & NS & NS & NS & \\
\hline \multicolumn{6}{|l|}{ Partenaires } \\
\hline P-S-J-G (Chine) & NS & NS & NS & NS & \\
\hline Bulgarie & & NS & & & \\
\hline Croatie & & NS & NS & & \\
\hline Hong-Kong (Chine) & & NS & NS & & \\
\hline Lituanie & NS & & NS & & \\
\hline Macao (Chine) & & & NS & & \\
\hline Monténégro & NS & NS & NS & & \\
\hline Qatar & NS & NS & & NS & \\
\hline Roumanie & NS & & & & \\
\hline Fédération de Russie & & NS & NS & & \\
\hline Singapour & & NS & NS & & \\
\hline Taipei chinois & NS & NS & & & \\
\hline Émirats arabes unis & NS & NS & & NS & \\
\hline Viet Nam & NS & & NS & & NS \\
\hline
\end{tabular}

Remarques : Résultats basés sur des modèles de régression logistique multi-niveaux, incluant des contrôles du cycle PISA (2012 ou 2015), du sexe des élèves, de leur statut socio-économique et de la langue qu'ils parlent en famille, ainsi que du profil socio-économique moyen des établissements. Seuls sont inclus dans l'analyse les pays/économies dans lesquels plus de $5 \%$ des élèves défavorisés sont résilients sur le plan scolaire.

Le caractère propice ou non à l'apprentissage du climat en classe est mesuré à l'aide des valeurs moyennes au niveau Établissement sur les indices PISA du climat de discipline en cours de sciences (PISA 2015) et en cours de mathématiques (PISA 2012).

Les pays et économies sont listés selon leur statut de pays membre ou partenaire de l'OCDE et par ordre alphabétique de leur nom en anglais.

Source : OCDE, Bases de données PISA 2012 et PISA 2015. 
Les pays ayant enregistré une hausse de leur pourcentage d'élèves résilients y sont parvenus soit en relevant le niveau moyen de performance (donc en améliorant la qualité de l'offre scolaire), soit en réduisant le niveau d'incidence du statut socio-économique sur la performance (donc en renforçant l'équité). Nombre des pays qui ont connu les progrès les plus rapides, tels que l'Allemagne, y sont parvenus grâce à la combinaison d'améliorations de la qualité des possibilités d'apprentissage pour tous les élèves et d'améliorations ciblant plus spécifiquement les élèves les plus défavorisés sur le plan socio-économique.

\section{Les élèves défavorisés réussissent lorsque leur établissement offre un climat discipliné et propice à l'apprentissage}

La probabilité pour les élèves défavorisés d'être résilients sur le plan scolaire varie non seulement entre les pays, mais aussi au sein même des système d'éducation, en fonction de l'établissement qu'ils fréquentent. Une analyse approfondie des données des enquêtes PISA 2012 et 2015 s'est concentrée sur un sous-ensemble de pays et d'économies où au moins $5 \%$ des élèves défavorisés sont résilients sur le plan scolaire. Elle a permis d'identifier certaines caractéristiques communes aux environnements scolaires dans lesquels les élèves défavorisés réussissent.

Dans la grande majorité des systèmes d'éducation à l'étude, la probabilité de résilience parmi les élèves défavorisés est plus forte dans les établissements où les élèves font part d'un bon climat de discipline, par rapport à ceux offrant un environnement plus indiscipliné, et ce même après contrôle des différences de profil socio-économique des élèves et des établissements, ainsi que d'autres caractéristiques individuelles associées à la résilience. Pouvoir suivre des cours dans un climat propice, permettant aux élèves de se concentrer et aux professeurs de dispenser leur enseignement au rythme qu'ils souhaitent, s'avère bénéfique pour tous les élèves, mais surtout pour les plus vulnérables d'entre eux. Une relation similaire s'observe avec le pourcentage d'élèves n'ayant séché aucune journée entière de cours durant les deux semaines précédant l'évaluation PISA, soit un autre indicateur du climat de l'établissement (propice dans ce cas).

À l'inverse, la probabilité de résilience parmi les élèves défavorisés n'est que faiblement liée au niveau de ressources humaines et matérielles disponibles dans leur établissement. II n'existe par exemple aucun lien entre le nombre d'ordinateurs à disposition des élèves et le pourcentage d'élèves résilients, après contrôle du climat de discipline et des caractéristiques contextuelles des élèves ; en outre, en moyenne, dans les pays à l'étude, le nombre d'activités extrascolaires n'est que faiblement lié à ce pourcentage. Dans nombre de pays, les élèves défavorisés sont plus susceptibles d'être résilients dans les établissements proposant de nombreuses activités extrascolaires (et disposant des ressources pour ce faire) ; toutefois, la relation entre la résilience des élèves et les activités extrascolaires est en moyenne faible, et même négative dans certains pays.

Dans la plupart des pays, il existe une relation positive entre la taille des classes et la résilience : les élèves défavorisés fréquentant des établissements où les classes ont des effectifs plus élevés tendent à mieux réussir sur le plan scolaire. Toutefois, cette relation peut aussi refléter le fait que les responsables politiques compensent le désavantage des élèves en réduisant la taille des classes.

Dans la plupart des pays, il n'existe aucune corrélation entre les indicateurs des ressources scolaires et le pourcentage d'élèves résilients ; il ne s'agit pas pour autant d'en déduire qu'il est inutile d'investir des moyens matériels dans l'éducation. Les résultats semblent plutôt indiquer que les ressources ne contribuent à la réussite scolaire des élèves défavorisés que lorsqu'elles améliorent les caractéristiques de l'environnement d'apprentissage en lien le plus direct avec leurs possibilités d'apprentissage.

\section{Pour conclure}

Les élèves issus de milieux socio-économiques défavorisés rencontrent souvent des obstacles qui les empêchent d'exprimer pleinement leur potentiel à l'école, et limitent de ce fait leur capacité à contribuer à la société et à profiter des possibilités d'apprentissage tout au long de la vie. L'un des principaux objectifs des systèmes d'éducation est de lever ces obstacles. En veillant à ce que les élèves défavorisés bénéficient d'un climat propice pour apprendre, et en enrichissant leur apprentissage par l'offre d'activités extrascolaires pertinentes, l'école peut être à l'avant-garde de l'action pour l'avènement d'une société plus inclusive et plus juste. 


\section{Pour tout complément d'information}

Contacter : Francesco Avvisati (Francesco.avvisati@oecd.org)

Consulter : Agasisti, T., F. Avvisati, F. Borgonovi et S. Longobardi, « Academic resilience: What schools and countries do to help disadvantaged students succeed in PISA », Documents de travail de l'OCDE sur l'éducation, n 167, http://dx.doi.org/10.1787/19939019.

Le mois prochain : Quel(s) aspect(s) professionnel(s) apportent le plus de satisfaction aux professeurs de sciences?

Ces travaux de recherche ont bénéficié du soutien et de la contribution de la Fondation Vodafone Allemagne au programme de travail PISA.

Cet ouvrage est publié sous la responsabilité du Secrétaire général de l'OCDE. Les opinions et les interprétations exprimées ne reflètent pas nécessairement les vues des pays membres de l'OCDE.

Ce document, ainsi que les données et cartes qu'il peut comprendre, sont sans préjudice du statut de tout territoire, de la souveraineté s'exerçant sur ce dernier, du tracé des frontières et limites internationales, et du nom de tout territoire, ville ou région.

Les données statistiques concernant Israël sont fournies par et sous la responsabilité des autorités israéliennes compétentes. L'utilisation de ces données par l'OCDE est sans préjudice du statut des hauteurs du Golan, de Jérusalem Est et des colonies de peuplement israéliennes en Cisjordanie aux termes du droit international.

Ce texte est disponible sous licence Attribution - Pas d'Utilisation Commerciale - Partage dans les Mêmes Conditions 3.0 Organisations Internationales (CC BY-NC-SA 3.0 IGO). Pour toute information spécifique quant à l'étendue et aux termes de la licence ainsi que d'une possible utilisation commercial de ce texte or pour toute usage de données PISA prière de consulter les Conditions d'utilisation à http://www.oecd.org/fr/conditionsdutilisation. 